\title{
Selenium-Enriched Agaricus Bisporus Mushroom Regulates Colonic Selenoprotein Expression in Rats Differently Under Thermoneutral and Hyperthermal Stress Conditions
}

\author{
Tebo Maseko, Frank Rowland Dunshea, Kate Howell, Ken Ng*
}

Faculty of Veterinary and Agricultural Sciences, University of Melbourne, Parkville, Victoria, Australia

*Corresponding Author: Ken Ng,Faculty of Veterinary and Agricultural Sciences, University of Melbourne, Melbourne, Victoria 3010, Australia.Tel:+61-3-903-531-41; E-Mail: ngkf@unimelb.edu.au

\begin{abstract}
Rats under thermoneutral conditions and fed mushroom-Se in dietary supplementation showed a significant fold increase $(\mathrm{P}<0.05)$ in mRNA expression relative to basal treatment (thermoneutral, control diet) of GPx-1 (2.98 \pm 0.82$)$, GPx-2 (1.8 \pm 1.4$)$, TrxR-1 (1.83 \pm 0.64$)$ and $\operatorname{SeP}(2.77 \pm 0.92)$. Supplementation with $\alpha$-tocopherol alone affected the expression of GPx-1 (1.83 \pm 0.82$)$ and SeP $(2.21 \pm 0.92)$. Rats subjected to heat stress and fed the control diet showed significant fold increase $(\mathrm{P}<0.05)$ in mRNA expression relative to the basal treatment of GPx-1 $(3.15 \pm 0.82)$ and SeP (1.93 \pm 0.92$)$. The expression of GPx-2 (3.2 \pm 1.4$)$ and $\mathrm{SeP}(4.23 \pm 0.92)$ in

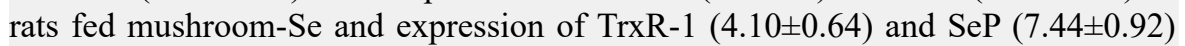
in rats fed $\alpha$-tocopherol alone were higher. Total colonic cellular GPx activity increased 3.8 fold in rats under thermoneutral conditions and fed the mushroom-Se diet compared to basal treatment. Heat stress had a negative impact on the colonic GPx activity which was recovered by mushroom-Se, but not $\alpha$-tocopherol.
\end{abstract}

Received Date: June 30, 2015

Accepted Date: October 15, 2015

Published Date: October21, 2015

Citation: $\mathrm{Ng}$, K., et al. Selenium-Enriched Agaricus Bisporus Mushroom Regulates Colonic Selenoprotein Expression in Rats Differently Under Thermoneutral and Hyperthermal Stress Conditions. (2015) Int J Food Nutr Sci 2(2):112-121.

DOI: $10.15436 / 2377-0619.15 .027$

Keywords: Se-enriched mushroom; Agaricus bisporus; Oxidative stress; Heat stress; Organic Se; Glutathione peroxidase-1; Glutathione peroxidase-2; Thioredoxin reductase-1;Selenoprotein P; Total GPx; $\alpha$-tocopherol

Abbreviations: ANOVA: Analysis of variance; CRC: Colorectal cancer; DDT: Dichlorodiphenyltrichloroethane; DMBA: 7, 12-dimethylbenz-[a]-anthracene; EDTA: Ethylenediamine tetra-acetic acid; GAPDH: Glyceraldehyde-3-phosphate dehydrogenase; GI:Gastrointestinal; GPx-1: Glutathione peroxidase 1; GPx-2: Glutathione peroxidase 2; LSD: Least Significant difference; MeSeCys: Methylselenocysteine; PCR: Polymerase Chain Reaction; ROS: Reactive oxygen species; RH: Relative humidity; RT: Room temperature; Se: Selenium; SeCys: Selenocysteine; SeMet: Selenomethionine; SeP: Selenoprotein P; TrxR-1: Thioredoxinreductase 1; NADPH: Nicotinamide adenine dinucleotide phosphate

\section{Introduction}

Selenium is an essential trace element and micronutrient required for various metabolic and physiological functions in mammals, and its significance in human health and disease prevention is well documented ${ }^{[1-3]}$. Selenium occurs in both organic and inorganic forms, with the organic Se present mainly as selenomethionine (SeMet), selenocysteine (SeCys) and methylselenocysteine (MeSeCys) while the inorganic Se exists mainly as selenite and selenate salts in foods ${ }^{[4-6]}$. The chemical form of Se is one of the determining factors of the element's bioavailability, metabolic processing and fate, biological efficacy and toxicity ${ }^{[7,8]}$. Moreover, it is the organic Se that has been linked to beneficial biological properties for good health and disease prevention ${ }^{[1,3]}$. This is supported 
by epidemiological and intervention studies that demonstrated higher Se intake above nutritional recommendations offer enhanced protection against cardiovascular and inflammations ailments $^{[3]}$, and reduced the incidence of colorectal, lung, prostate and liver cancers ${ }^{[9,10]}$.

Human intervention studies have demonstrated that high dietary Se supplementaion at $200 \mu \mathrm{g}$ Se per day in the form of selenised yeast decreased the incidence and mortality of several types of cancers (prostate, colorectal and lung) by $50 \%$ over a period of ten years ${ }^{[1]}$. Furthermore, animal studies have revealed that dietary Se supplementation with selenised $A$. bisporus mushroom at $1 \mu \mathrm{g} \mathrm{Se} / \mathrm{g}$ feed reduced 7, 12-dimethylbenz-[a]-anthracene (DMBA) induced mammary DNA adducts and decreased the incidence of anti-3, 4-dihydrodiol-1, 2-epoxide-deoxyguanosine adducts in DMBA challenged rats ${ }^{[10]}$. More recent studies have also demonstrated the protective effect of organic Se against diseases in the form of selenised milk proteins at $1 \mu \mathrm{g} \mathrm{Se} / \mathrm{g}$ feed, which suppressed aberrant crypt foci, $\mathrm{K}$-ras mutations and colorectal oncogenesis in azoxymethane challenged mice ${ }^{[11]}$

The biological efficacy and anti-carcinogenic benefits of organic Se are believed to come about through the expression of selenoamino acids containing selenoproteins and selenoenzymes, which are involved in mitigating cellular oxidative stress by inactivating cellular oxidants such as reactive oxygen species $(\mathrm{ROS})^{[2,12-14]}$. The selenoenzymes, which contain catalytic selenocysteine at the active site, include the glutathione peroxidases (GPxs) and thioredoxinreductases (TrxRs) and are the major cellular antioxidant enzymes ${ }^{[2,15-17]}$.

Colorectal cancer (CRC) is reportedly the third most frequent cause of cancer mortality and the fourth most common cause of death in the world ${ }^{[18]}$. Evidence suggested that oxidative stress induced by heat or other factors is characterized by the accumulation of ROS, and that they are significant contributing factors in gastrointestinal tract dysfunction and the pathogenesis of gastrointestinal tract ailments such as inflammatory bowel disease, fibrosis, ulcerative colitis and $\mathrm{CRC}^{[13,19]}$. The over production of ROS causes epithelial cells damage, reduced intestinal barrier function and integrity, and increases CRC risks ${ }^{[19,20]}$.

Human epidemiological studies have shown an inverse relationship between organic Se intake and overall cancer risk, including $\mathrm{CRC}^{[1]}$. GPxselenoenzyme isoforms including cytosolic GPx-1 which is expressed in almost all tissues, and gastrointestinal tract specific GPx-2, are the major selenoenzymes that play a significant role in the antioxidant defense of the gastrointestinal tissues against oxidative injury ${ }^{[13,21]}$. Several animal and human studies have demonstrated an up-regulation in expression of selenoproteins and selenoenzymes following dietary Se supplementation. Hu et al. ${ }^{[22]}$ and Uglietta, et al. ${ }^{[23]}$ showed an up-regulation in mRNA expression and activity of colonic GPxs following dietary Se supplementation with yeast-Se in mouse and pig respectively. Furthermore, human clinical trials showed an increase in expression of rectal GPxs and selenoprotein $\mathrm{P}$ (SeP) following dietary Se supplementation with dairy-Se and yeast-Se ${ }^{[24]}$.

We have previously shown that Se-enriched A. bisporus mushroom cultivated by irrigation of growth compost with sodium selenite contains organic Se mainly as selenocysteine rich selenoproteins ${ }^{[25]}$. We also demonstrated that dietary Se supplementation with the mushroom-Se up-regulated colonic GPx-
1 activity and mRNA expression of GPx-1 and GPx-2 in rats ${ }^{[26]}$. We have also demonstrated the protective effects of dietary Se supplementation with the same mushroom-Se against gut barrier dysfunction as indicated by reduced ileum permeability during hyperthermally induced oxidative stress in rat ${ }^{[27]}$.

In this study, we evaluated the effects of dietary Se supplementation with mushroom-Se and with or without additional supplementation with $\alpha$-tocopherol on the regulation of the expression of colonic selenoproteins GPx-1, GPx-2, TrxR-1 and $\mathrm{SeP}$ in hyperthermally induced oxidative stress in rat. The study sought to determine how hyperthermally induced oxidative stress regulated the different colonic selenoproteins of relevance to anticancer function in rats placed on a high Se diet in the form of mushroom-Se, and whether additional supplementation with $\alpha$-tocopherol enhanced the Se effect as previously reported by others $^{[28]}$.

\section{Experimental Section \\ Se-Enriched and Non-Se Enriched A. Bisporus Mushroom}

Se-enriched $A$. bisporus (white button mushroom) was cultivated using grower kits comprised of growth compost inoculated with $A$. bisporus spawn, supplied by a commercial mushroom producer (Mushroom Exchange Pty. Ltd., Mernda, Victoria, Australia) and irrigation with sodium selenite solution as described elsewhere ${ }^{[26]}$. Non-Se enriched $A$. bisporus was also cultivated under normal mushroom growing conditions without irrigation with sodium selenite but water only. Mushroom caps were harvested from both mushroom types, frozen at $-80^{\circ} \mathrm{C}$, and freeze-dried. The lyophilised caps were then grounded into fine powders using a commercial blender and stored at RT in moisture free sealable packs until required for preparation of rat feeds.

\section{Diets: Preparation of Control, Se-Enriched, A-Tocophero- land Se-Enriched + A-Tocopherol Diets}

Rat feed was prepared in the form of pellets by Specialty Feeds Co (Glen Forrest, Western Australia). Four diet types including the control were prepared. The (i) low $\mathrm{Se}+$ low $\alpha$-tocopherol diet serving as the control diet was prepared by supplementing $10 \mathrm{~kg}$ of a low Se rodent feed formulation AIN 93G (composition detailed in ${ }^{[27]}$ ) with $20 \mathrm{~g}$ control (non-Se enriched) mushroom caps (containing $2.22 \mu \mathrm{g} \mathrm{Se} / \mathrm{g}$ dried caps) with no $\alpha$-tocopherol supplementation to give a final Se content of 0.12 $\mu \mathrm{g} \mathrm{Se} / \mathrm{g}$ feed. The (ii) high $\mathrm{Se}+$ low $\alpha$-tocopherol diet was prepared by supplementing $10 \mathrm{~kg}$ of AIN 93G with $142 \mathrm{~g}$ of Se-enriched mushroom caps (containing $62.20 \mu \mathrm{g} \mathrm{Se} / \mathrm{g}$ dried caps) with no $\alpha$-tocopherol supplementation to give a final Se content of $1 \mu \mathrm{g} \mathrm{Se} / \mathrm{g}$ feed. The (iii) low Se + high $\alpha$-tocopherol diet was similarly prepared as the control diet but with the addition of $\alpha$-tocopherol to give a final Se and $\alpha$-tocopherol contents of 0.12 $\mu \mathrm{g} \mathrm{Se} / \mathrm{g}+300 \mu \mathrm{g} \alpha$-tocopherol/g feed. The (iv) high Se + high $\alpha$-tocopherol diet were similarly prepared as the high Se diet but with the addition of $\alpha$-tocopherol to give a final Se and $\alpha$-tocopherol contents of $1 \mu \mathrm{g} \mathrm{Se} / \mathrm{g}+300 \mu \mathrm{g} \alpha$-tocopherol $/ \mathrm{g}$ feed.

\section{Animals}

A total of sixty four 9 weeks old Sprague Dawley male rats weighing 302-426 g obtained from a colony without known adventitious viruses, mycoplasma, enteric pathogenic bacteria and parasites, were purchased from the Monash University 
Animal Services, Melbourne, Australia. All 64 male rats were healthy. The animal experiment protocols were approved by the Melbourne School of Land \& Environment Research Animal Ethics Committee, University of Melbourne (ethics approval no. 1312820.1). Rats were randomly divided into 4 experimental dietary groupsand housed 2 per cage. The rats were housed in an air-conditioned, temperature controlled animal facility with a 12 $\mathrm{h}$ light-dark cycle at $21^{\circ} \mathrm{C}$. Rats were given free access to food and water at all times.

\section{Animal Feeding}

Rats were randomly assigned to 4 experimental diets in a 2 (Se levels) x 2 ( $\alpha$-tocopherol levels) factorial design: (i) Control diet of low $\mathrm{Se}+$ low $\alpha$-tocopherol; (ii) High Se diet of high Se + low $\alpha$-tocopherol; (iii) High $\alpha$-tocopherol diet of low $\mathrm{Se}+$ high $\alpha$-tocopherol; (iv) High Se and high $\alpha$-tocopherol diet of high $\mathrm{Se}+$ high $\alpha$-tocopherol. All rats were initially acclimatized with free access to the control diet and water for one week before the animals were given their respective diets and water ad libitum for a further period of 3 weeks. Body weights of the animals were recorded at the start of the experiment and continued weekly to monitor their growth which was normal. Their food intake and behavior were also monitored throughout. These were also normal.

\section{Acute Heat Stress Protocol}

After 21 days of feeding of the allocated diets, rats from each diet were randomly allocated to thermoneutral and acute heat stress treatment groups in a $2 \times 2 \times 2$ factorial design (2 levels Se x 2 levels $\alpha$-tocopherol x 2 levels temperature) and their body weights determined. Rats in the thermoneutral treatments were exposed to an ambient temperature of $21^{\circ} \mathrm{C}$ and a relative humidity $(\mathrm{RH})$ of $40 \%$ and had their feed removed but allowed free access to water for a period of $90 \mathrm{~min}$. Rats exposed to acute heat stress were housed individually in cages, exposed to $40^{\circ} \mathrm{C}$ and $21 \%$ relative humidity (RH) and also had their feed removed and allowed free access to water for a $90 \mathrm{~min}$ period in a temperature controlled room. Both rat experimental groups accessed drinking water at a temperature of $20.4^{\circ} \mathrm{C}$. Preliminary observation of rats indicated that they can tolerate $40^{\circ} \mathrm{C}$ and $21 \%$ RH conditions for up to $90 \mathrm{~min}$ but beyond that time point distress symptoms such as hyper ventilation and lethargy sets in, thus the thermoneutral and acute heat stress condition was limited to $90 \mathrm{~min}$ exposure. The rats on the control diet of low $\mathrm{Se}+$ low $\alpha$-tocopherol accessed drinking water more frequently compared to other rat groups under heat stress.

Animals from both treatments were monitored every 10 min over the $90 \mathrm{~min}$ treatment duration for rectal temperatures using a temperature probe (Vicks Speed-Read Digital thermometer, $10 \mathrm{~mm} \times 3 \mathrm{~mm}$ probe) and heart rates using a stethoscope (as beats per minute: bpm). Lubricant was used to aid thermometer probe insertion into the rectum. At the end of the treatments, rats were allowed to rest for $20 \mathrm{~min}$ at RT before being anaesthetized and sacrificed for tissue excision.

\section{Animal Euthanasia and Tissue Excision (Colon)}

Animals were killed with an initial step of a single intra-peritoneal injection of ketamine and xylazine mix to anaesthetize and an overdose of the ketamine and xylazine mix as the final euthanasia step. Colon tissues were recovered from each rat, and a portion of each tissue was separately placed in RNAlater ${ }^{\circledR}$ solution at $4^{\circ} \mathrm{C}$ for $24 \mathrm{~h}$ before storage at $-80^{\circ} \mathrm{C}$ until real time PCR analysis. The remaining portion of the colon tissue from the rat was rapidly frozen in liquid $\mathrm{N} 2$ and stored at $-80^{\circ} \mathrm{C}$ for GPx-1 activity assay.

\section{Gene Expression \\ RNA Isolation}

Total RNA was extracted from RNAlater ${ }^{\circledR}$ solution stabilized colon tissues $(30 \mathrm{mg}$ ) using a commercial kit, QIAGEN RNeasy® Mini Kit (QIAGEN, Victoria, Australia), and the extraction was performed in triplicates for each rat colon tissue. The quality (purity) and concentration of total RNA extracted was determined using NanoDrop ${ }^{\circledR}$ ND-1000 UV-Vis spectrophotometer by measuring the absorbance at $\lambda 260 \mathrm{~nm}$ and 280 $\mathrm{nm}$ and determining the 260:280 absorbance ratios. Pure RNA has an A260:A280 ratio of 1.9-2.1, and the extracted RNA samples from the rats' colon tissues had A260: A280 ratios between 1.94 and 2.13 (data not shown).

\section{cDNA Synthesis}

The BIO-RAD iScript ${ }^{\circledR}$ Select cDNA Synthesis Kit (NSW, Australia) was used to synthesize the first strand cDNA $(20 \mu \mathrm{L})$ from $0.3 \mu \mathrm{g}$ total RNA from each colon sample. The cDNA product was diluted 1:30 with nuclease-free water and used for real-time quantitative PCR.

\section{Real-Time Quantitative PCR}

Real-time quantitative PCR of GPx-1, GPx-2, SeP, and TrxR-1 genes was carried on an $\mathrm{iQ}^{\mathrm{TM}} 5$ Multicolor Real-Time PCR icycler® Detection System (BIORAD, NSW, Australia). Oligonucleotide primers were designed using Primer 3 software v.0.4.0 (Bioinformatics Methods and Protocols: Methods in Molecular Biology, Humana Press, Totowa, NJ, USA) based on sequences obtained from the Genbank database. The primers were optimized and validated by conventional PCR of cDNA (data not shown). The PCR reagents for quantitative analysis were contained in the iQ SYBR Green Supermix kit from BIORAD. The PCR reactions were performed in a final volume of $20 \mu \mathrm{L}$ containing $6 \mu \mathrm{L}$ of diluted cDNA (1:30, v/v) and $10 \mu \mathrm{L}$ iQ SYBR Green Supermix. Primer concentrations in the reaction mix for each gene was $250 \mathrm{nM}$ for both the sense and antisense primer pairs. Nuclease free water was used to make up to the final volume.

The cycling PCR reaction for each sample started with an initial hot start of $95^{\circ} \mathrm{C}$ for $3 \mathrm{~min}$ as the initial denaturation step of 1 cycle, followed by 45 cycles at $95^{\circ} \mathrm{C}$ for $30 \mathrm{~s}$ (denaturation), $60^{\circ} \mathrm{C}$ for $30 \mathrm{~s}$ (annealing), $72^{\circ} \mathrm{C}$ for $30 \mathrm{~s}$ (extension) and completed with a final extension step at $95^{\circ} \mathrm{C}$ for $1 \mathrm{~min}$. The specificity of the PCR reaction (product) was demonstrated by melting curve analysis post PCR reactions; which showed only one peak present for all PCR products of GPx-1, GPx-2, SeP, and TrxR-1 test genes and the glyceraldehyde-3-phosphate dehydrogenase, GAPDH, reference gene. A non-template (without cDNA) reaction was included with each PCR run as a negative control. The real-time quantitative PCR assay was optimized by running serial dilutions of cDNA template and using the results to generate a standard curve. The linear regression line and the coefficient of determination $\left(\mathrm{R}^{2}\right)$ of the standard curve were used to evaluate whether the qPCR assay was optimized. Amplifica- 
tion efficiency (E) of primer pair for each gene was calculated from the slope of the standard curve. Relative gene expression for each target gene, in the test and control samples using reference GAPDH gene as a normalizer was determined. The cycle threshold (CT) values for each target gene were normalized with the reference gene $\mathrm{GAPDH}^{[29]}$ for both test and control samples, and the Pfaffl method of relative quantification normalized to a reference gene was used to calculate the relative gene expression of the target genes in the colon samples (BIORAD Real-Time PCR Applications Guide-Gene Expression).

Total colonic cellular glutathione peroxidase (GPx) activity assay

Glutathione peroxidase activity was measured in rat colon using a Glutathione Peroxidase Cellular Activity Assay Kit (Sigma-Aldrich, cat no CGP1, NSW, Australia). The colon was cut open and the mucosa layer scraped off. The colon was then placed in $1 \mathrm{ml}$ of cold $\left(4^{\circ} \mathrm{C}\right) 50 \mathrm{mMTris}-\mathrm{HCl}$ buffer $(\mathrm{pH} 8)$ containing $0.5 \mathrm{mM} \mathrm{EDTANa}_{2}$ in a $2 \mathrm{~mL}$ tube containing 7 ceramic beads $(2.8 \mathrm{~mm}$ diameter ceramic beads, cat no 1311450 , Gene Works, Australia). The tube was placed in a rotor-stator tissue disruptor bead beater (MB8 model, BioSpec Products, DainTree Scientific Pty, Australia) for 5 min to lyse and homogenize the tissue and the homogenate quickly placed in an ice bath to chill. The homogenate was then centrifuged at 14,000 g (Beckman Benchtop Centrifuge, Allegra X-22 series, Gladesville, Australia) for $35 \mathrm{~min}$ and the total solubilized colonic GPx was collected in the supernatant.

Protein concentration in the $50 \mu \mathrm{L}$ of the supernatant was determined in triplicate using the Bradford protein assay kit (cat no. B6916-500, Sigma Aldrich, NSW, Australia). Total GPx activity in $50 \mu \mathrm{L}$ of the supernatant was determined in triplicate by adding $950 \mu \mathrm{L}$ of reaction solution containing $50 \mathrm{mM}$ TrisHCl buffer (pH 8), $0.5 \mathrm{mM}$ EDTA, $5 \mathrm{mM}$ NADPH, $42 \mathrm{mM}$ reduced glutathione, 10 units $/ \mathrm{mL}$ glutathione reductase and 30 $\mathrm{mM}$ tert-butyl hydroperoxide as the substrate. A glutathione peroxidase standard stock solution (100 unit $/ \mathrm{ml}$ supplemented with $1 \mathrm{mg} / \mathrm{mL} \mathrm{IgG}$ and $1 \mathrm{mM}$ DDT) was used as a positive activity control. The decrease in NADPH was measured every $30 \mathrm{~s}$ over a $10 \mathrm{~min}$ time period by absorbance at $\lambda 340 \mathrm{~nm}$ using a UV-Vis spectrophotometer (Multiskan spectrophotometer, Thermo Electron, Thermo Fishers Scientific, USA). Oxidation of NADPH to $\mathrm{NADP}^{+}$was linear over the $10 \mathrm{~min}$ time interval. One unit of glutathione peroxidase is defined as the enzyme reaction that causes the formation of $1 \mu \mathrm{mol}$ of $\mathrm{NADP}^{+}$from NADPH per min in the presence of $40 \mathrm{mM}$ reduced glutathione, $9.5 \mathrm{unit} / \mathrm{mL}$ glutathione reductase and $28.5 \mathrm{mM}$ tert-butyl hydroperoxide. GPxactivity was expressed as $\mathrm{mU} / \mathrm{mg}$ protein.

\section{Statistical Analysis}

The mean of triplicate determinations was used to calculate the group means and uncertainty as standard error. Uncertainties were presented as pooled standard errors of differences of means obtained from statistical analyses by three-factor analysis of variance (ANOVA); 2 (levels of Se) x 2 (levels of $\alpha$-tocopherol) $x 2$ (levels of temperature), with a subsequent multiple comparison test by Fisher's Protected Least Significant Difference (LSD) test using GenStat $\left(14^{\text {ed }}\right)$. Statistical significance was defined as $\mathrm{P}$ value less than $0.05(\mathrm{P}<0.05)$ for groups means and letters subscripts ${ }^{\mathrm{a}, \mathrm{b}, \mathrm{c}}$ used to indicate multiple comparisons be- tween groups. Uncertainty of group means was reported to 2 significant figures according to the European Analytical Chemist guidelines $^{[30]}$.

\section{Results}

\section{Glutathione Peroxidase-1 mRNA Expression}

The expression level of GPx-1, GPx-2, TRx-1 or SeP mRNA of rats kept under thermoneutral conditions and fed the control diet (low $\mathrm{Se}+$ low $\alpha$-tocopherol) is defined as the basal level and is set at an arbitrary level of 1 (Figure 1,2). Differences in gene expressions between rats fed with control feed and $\mathrm{Se}$ supplemented feed were measured.
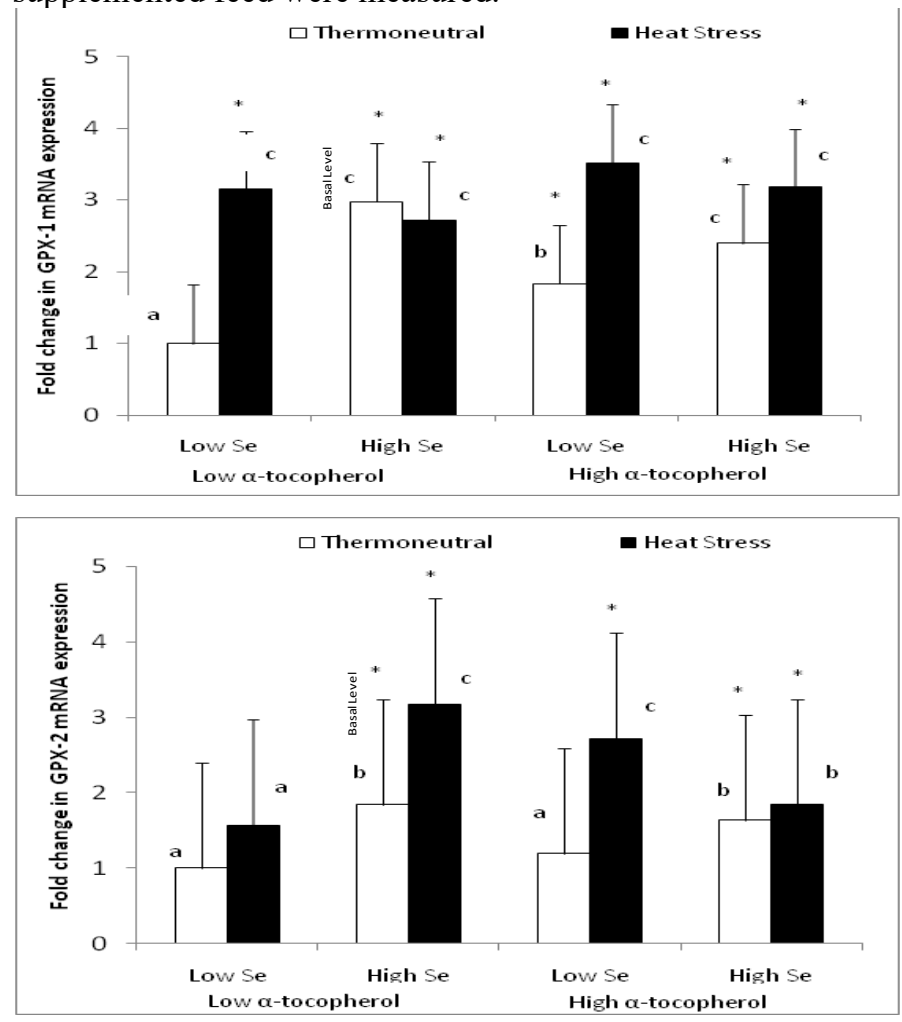

Figure 1: Effect of dietary mushroom Se supplementation on colon (A) GPx-1 and (B) GPx-2 mRNA expressions from rats exposed to thermoneutral $\left(\mathrm{T} 21^{\circ} \mathrm{C}, 40 \%\right.$ R.H) or acute heat stress conditions $\left(\mathrm{T} 40^{\circ} \mathrm{C}, 21 \%\right.$ R.H., $90 \mathrm{~min}$ exposure). mRNA levels were measured in triplicate colon tissues excised from the colon of each rat fed the control diet (low Se + low $\alpha$-tocopherol; $0.12 \mu \mathrm{g} \mathrm{Se} / \mathrm{g}$ feed), high Se diet (high Se + low $\alpha$-tocopherol; 1 $\mu \mathrm{g} \mathrm{Se} / \mathrm{g}$ feed), high $\alpha$-tocopherol diet (low Se + high $\alpha$-tocopherol; $0.12 \mu \mathrm{g} \mathrm{Se} / \mathrm{g}$ feed $+300 \mu \mathrm{g} \alpha$-tocopherol/g feed) or high $\mathrm{Se}+$ high $\alpha$-tocopherol diet (high $\mathrm{Se}+$ high $\alpha$-tocopherol; $1 \mu \mathrm{g}$ $\mathrm{Se} / \mathrm{g}$ feed $+300 \mu \mathrm{g} \alpha$-tocopherol/g feed), and expression level normalized against the glyceraldehyde-3-phosphate dehydrogenase (GAPDH) reference gene. Fold change $(n=8)$ in GPx-1 and GPx-2 mRNA levels was calculated relative to a basal level from thermoneutral rats fed the control diet, which was set at an arbitrary expression level of 1 .

Error bar indicates pooled standard errors of differences of means ( \pm 0.82 for GPx-1 and 1.4 for GPx-2).

*Mean values statistically significantly different from the control treatment (basal level) ( $\mathrm{P}<0.05$ in 3 -factor ANOVA).

a,b,c Indicate significant differences $(\mathrm{P}<0.05)$ of means in the multiple comparisons between groups in the Fisher's Protected LSD multiple comparison test. 
Dietary supplementation with mushroom Se at $1 \mu \mathrm{g}$ $\mathrm{Se} / \mathrm{g}$ feed and with $\alpha$-tocopherol at $300 \mu \mathrm{g} / \mathrm{g}$ feed and acute heat stress all significantly increased $(\mathrm{P}<0.05)$ colonic GPx-1 mRNA expression in rats (Figure 1A). There was an interaction between dietary Se and heat stress treatment such that GPx-1 mRNA expression was increased $(\mathrm{P}<0.05)$ during heat stress for rats not supplemented with Se. Thus, rats fed the high Se diet and kept under thermoneutral conditions displayed a significant increase $(\mathrm{P}<0.05)$ in $\mathrm{GPx}-1$ expression similar to the effect of heat stress in the low Se diet (2.98 and 3.15 fold increase above the basal level, respectively). A combination of mushroom-Se with $\alpha$-tocopherol in the high $\mathrm{Se}+$ high $\alpha$-tocopherol diet and rats maintained under thermoneutral condition produced no additional significant increase $(\mathrm{P}<0.05)$ in $\mathrm{GPx}-1$ mRNA expression $(2.40$ fold increase) (Figure 1A).

Acute heat stress significantly increased $(\mathrm{P}<0.05)$ GPx-1 mRNA expression across all diets relative to the control treatment (basal level), with rats on the high $\alpha$-tocopherol diet displaying the highest GPx-1 expression (3.52 fold increase). Although rat groups placed on the high Se diet and maintained under thermoneutral conditions or exposed to heat stress both displayed a significant increase $(\mathrm{P}<0.05)$ in GPx-1 mRNA expression relative to the basal level, there was however no additional induction $(\mathrm{P}<0.05)$ in GPx-1 mRNA expression as a result of hyperthermia (2.98 and 2.72 fold increase, respectively) (Figure 1A). Furthermore, there were no significant differences $(\mathrm{P}<0.05)$ in $\mathrm{GPx}-1$ expression amongst all diets under acute heat stress conditions.

\section{Glutathione Peroxidase-2 mRNA Expression}

GPx-2 mRNA expression was significantly increased $(\mathrm{P}<0.05)$ by dietary supplementation with mushroom Se and acute heat stress but not with $\alpha$-tocopherol under thermoneutral conditions (Figure 1B). There was also an interaction between Se and $\alpha$-tocopherol on the GPx-2 gene. Supplementation with $\alpha$-tocopherol to the high Se diet did not show any further significant enhancement $(\mathrm{P}<0.05)$ of GPx-2 expression for both thermoneutral and heat stress conditions, as both diets stimulated the level of GPx-2 similarly with no further expression under heat stress (1.63 and 1.84 fold increase, respectively) (Figure 1B). However, unlike GPx-1, rats placed on the high $\alpha$-tocopherol diet and subjected to heat stress treatment significantly increased $(\mathrm{P}<0.05) \mathrm{GPx}-2$ expression further than those on the control diet and exposed to heat stress treatment (2.72 and 1.60 fold increase, respectively). Furthermore, in contrast with GPx-1, rats fed the high Se diet and subjected to heat stress treatment also significantly increased $(\mathrm{P}<0.05) \mathrm{GPx}-2$ expression further than those exposed to heat stress treatment alone in the control diet (3.17 and 1.60 fold increase, respectively) (Figure 1B). Supplementation with $\alpha$-tocopherol (high $\alpha$-tocopherol) in the high $\mathrm{Se}$ diet under both thermoneutral and heat stress conditions did not further enhance the effect of Se on GPx-2 mRNA expression.

\section{Thioredoxin Reductase-1 mRNA Expression}

TrxR-1 mRNA expression was increased $(\mathrm{P}<0.05)$ by dietary Se, dietary $\alpha$-tocopherol and acute heat stress (Figure 2A). However, there were interactions between dietary mushroom Se and $\alpha$-tocopherol, and between $\alpha$-tocopherol and heat stress such that heat stress increased TrxR-1 expression to a much greater level in rats placed on the high $\alpha$-tocopherol diet than those fed the high Se diet (4.10 and 2.21 fold increase, respectively). Rats kept under thermoneutral conditions and fed the high Se diet also significantly increased $(\mathrm{P}<0.05)$ TrxR-1 expression (1.83 fold increase), however there was no significant increase $(\mathrm{P}<0.05)$ in TrxR-1 expression for rats on the high $\alpha$-tocopherol diet or the high $\mathrm{Se} / \alpha$-tocopherol combination diets (1.39 and 1.19 fold increase, respectively). Furthermore, supplementation with $\alpha$-tocopherol to the high Se diet did not further enhance $(\mathrm{P}<0.05)$ the expression of TrxR-1 for rats exposed to acute heat stress compared to supplementation with the high Se diet only (2.28 and 2.21 fold increase, respectively) (Figure 2A).
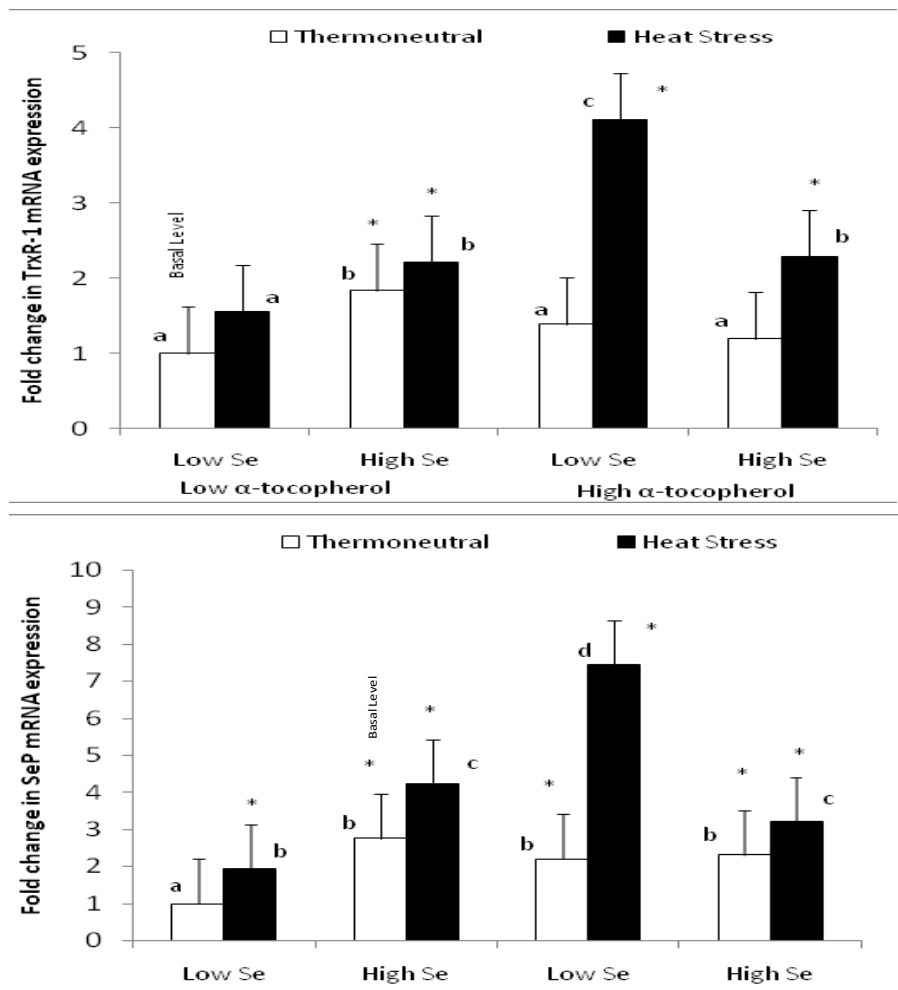

Figure 2: Effect of dietary mushroom Se supplementation on colon (A) TrxR-1 and (B) SeP mRNA expression from rats exposed to thermoneutral $\left(\mathrm{T} 21{ }^{\circ} \mathrm{C}, 40 \%\right.$ R.H) or acute heat stress conditions $\left(\mathrm{T} 40^{\circ} \mathrm{C}, 21 \%\right.$ R.H., 90 min exposure). mRNA levels were measured in triplicate colon tissues excised from the colon of each rat fed the control diet (low $\mathrm{Se}+$ low $\alpha$-tocopherol; $0.12 \mu \mathrm{g} \mathrm{Se} / \mathrm{g}$ feed) : high Se diet (high Se + low $\alpha$-tocopherol; $1 \mu \mathrm{g} \mathrm{Se} / \mathrm{g}$ feed) : high $\alpha$-tocopherol diet (low $\mathrm{Se}+$ high $\alpha$-tocopherol; $0.12 \mu \mathrm{g} \mathrm{Se} / \mathrm{g}$ feed $+300 \mu \mathrm{g} \alpha$-tocopherol/g feed) or high $\mathrm{Se}+$ high $\alpha$-tocopherol diet (high Se + high $\alpha$-tocopherol; $1 \mu \mathrm{g} \mathrm{Se} / \mathrm{g}$ feed $+300 \mu \mathrm{g} \alpha$-tocopherol/g feed), and expression level normalized against the glyceraldehyde-3-phosphate dehydrogenase (GAPDH) reference gene. Fold change $(n=8)$ in TrxR-1 and SeP mRNA levels was calculated relative to a basal level from thermoneutral rats fed the control diet, which was set at an arbitrary expression level of 1.

Error bar indicates pooled standard errors of differences of means ( \pm 0.64 for TrxR-1 and \pm 0.92 for $\mathrm{SeP}$ ).

* Mean values statistically significantly different from the control treatment (basal level) $(\mathrm{P}<0.05$ in 3-factor ANOVA).

a,b,c Indicate significant differences $(\mathrm{P}<0.05)$ of means in the multiple comparisons between groups in the Fisher's Protected LSD multiple comparison test.

\section{Selenoprotein P mRNA Expression}

It was observed that dietary $\mathrm{Se}, \alpha$-tocopherol supplementation and heat stress significantly increased $(\mathrm{P}<0.05)$ the expression of SeP mRNA (Figure 2B). There were interactions 
between dietary $\alpha$-tocopherol, selenium and heat stress $(\mathrm{P}<0.05)$. Similar to TrxR-1 expression, acute heat stress increased SeP expression to a much higher level $(\mathrm{P}<0.05)$ for rats that fed the high $\alpha$-tocopherol diet compared to rats fed the high $\mathrm{Se} / \alpha$ tocopherol diet combination (7.44 and 3.22 fold increase, respectively) (Figure 2B). However, there was no significant difference $(\mathrm{P}<0.05)$ in the level of colonic SeP expression between heat stress rats fed the high Sedietand the high Se/ $\alpha$-tocopherol combination diet (4.23 and 3.22 fold increase, respectively). In contrast with TrxR-1, rats placed under thermoneutral conditions and fed the high $\alpha$-tocopherol diet displayed a significant increase $(\mathrm{P}<0.05)$ in SeP expression above basal level $(2.21$ fold increase) and SeP expression for the high $\alpha$-tocopherol diet was not significantly different from the high $\mathrm{Se} / \alpha$-tocopherol combination diets under thermoneutral conditions (2.21 and 2.32 fold increase, respectively). Although dietary supplementation with mushroom Se produced a significant increase $(\mathrm{P}<0.05)$ in $\mathrm{SeP}$ expression relative to the control diet, there was no enhancement of the Se effect by $\alpha$-tocopherol supplementation under thermoneutral conditions (2.77 and 2.32 fold increase) (Figure 2B).

\section{Total Colonic Cellular Glutathione Peroxidase Activity}

Total GPx activity was measured in rat colon and the activity from rats kept under thermoneutral conditions and fed the control diet (low $\mathrm{Se}+$ low $\alpha$-tocopherol) defined as the basal total colonic GPxactivity level, which was $9.7 \pm 1.5 \mathrm{U} / \mathrm{mg}$ protein (Figure 3).

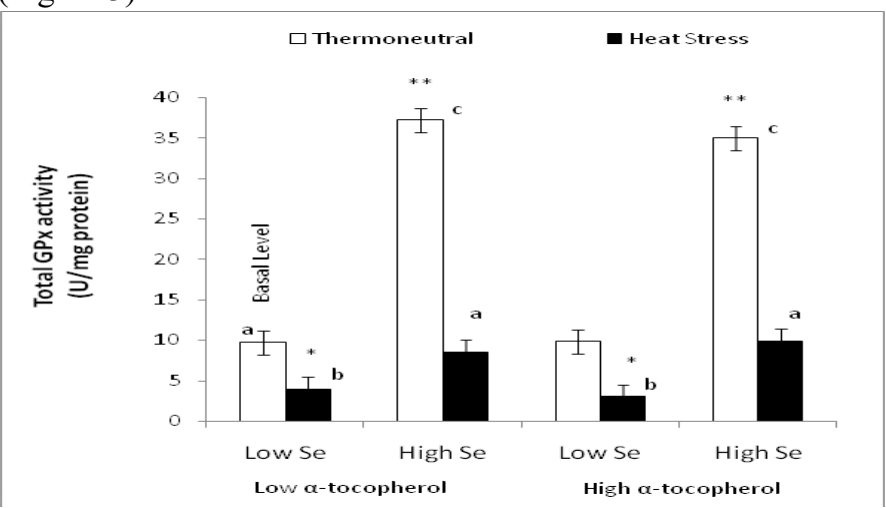

Figure 3: Effect of dietary mushroom Se supplementation on total colon GPx activity from rats exposed to thermoneutral $\left(\mathrm{T} 21^{\circ} \mathrm{C}, 40 \% \mathrm{RH}\right)$ or acute heat stress conditions $\left(\mathrm{T} 40^{\circ} \mathrm{C}, 21 \%\right.$ R.H., 90 min exposure). Total GPx activity were measured in triplicate colon tissues excised from the colon of each rat $(\mathrm{n}=8)$ fed the control diet (low $\mathrm{Se}+$ low $\alpha$-tocopherol; $0.12 \mu \mathrm{g} \mathrm{Se} / \mathrm{g}$ feed), high Se diet (high Se + low $\alpha$-tocopherol; $1 \mu \mathrm{g} \mathrm{Se} / \mathrm{g}$ feed), high $\alpha$-tocopherol diet (low Se + high $\alpha$-tocopherol; $0.12 \mu \mathrm{g} \mathrm{Se} / \mathrm{g}$ feed $+300 \mu \mathrm{g} \alpha$-tocopherol $/ \mathrm{g}$ feed) or high Se + high $\alpha$-tocopherol diet (high Se + high $\alpha$-tocopherol; $1 \mu \mathrm{g} \mathrm{Se} / \mathrm{g}$ feed +300 $\mu \mathrm{g} \alpha$-tocopherol/g feed).

Error bar indicates pooled standard error of differences of means $( \pm 1.55$ $\mathrm{U} / \mathrm{mg}$ protein).

*,** Mean values statistically significantly different (lower or higher) from the control treatment (basal level) ( $\mathrm{P}<0.05$ in 3-factor ANOVA).

a,b,c Indicate significant differences $(\mathrm{P}<0.05)$ of means in multiple comparisons between groups in the Fisher's Protected LSD multiple comparison test.

Total GPx activity in the colon significantly increased $(\mathrm{P}<0.05)$ by 3.8 fold increase above the basal level following dietary supplementation with Se $(37.2 \pm 1.5 \mathrm{U} / \mathrm{mg}$ protein) but remained unchanged $(\mathrm{P}<0.05)$ with dietary $\alpha$-tocopherol sup- plementation under thermoneutral conditions. (Figure 3). There was no further enhancement of GPx activity with the supplementation of $\alpha$-tocopherol to the high Se diet for rats under thermoneutral conditions (34.9 U/mg protein).

GPx activity was markedly reduced for rats subjected to acute heat stress and fed the control and the high $\alpha$-tocopherol diets (3.95 and $3.04 \mathrm{U} / \mathrm{mg}$ protein, respectively), indicating the failure of $\alpha$-tocopherol to protect the colon from loss ofGPx activity due to heat stress at low Se levels. However, dietary Se supplementation restored $(\mathrm{P}<0.05)$ GPx activity under heat stress to the same level as that of the basal level under thermoneutral conditions $(8.6 \pm 1.5 \mathrm{U} / \mathrm{mg}$ protein) (Figure 3). Furthermore, although the high $\mathrm{Se} / \alpha$-tocopherol combination diet also restored $(\mathrm{P}<0.05)$ colonic GPx activity to basal level under heat stress, there was no further improvement in GPx activity relative to the effect of high Se alone $(8.6$ and $9.95 \mathrm{U} / \mathrm{mg}$ protein, respectively.

\section{Discussion}

The study observed an up-regulation in the expression of all colonic selenoproteins examined following dietary Se supplementation under both thermoneutral and acute heat stress conditions. However, it was observed that the effects of dietary Se and $\alpha$-tocopherol supplementation and acute heat stress regulated the colonic selenoproteins uniquely. During acute heat stress, it was observed that SeP was expressed at the highest level compared to all selenoproteins, followed by TrxR-1 and then the GPxs. Dietary supplementation with Se but not $\alpha$-tocopherol increased colonic total GPx activity under thermoneutral conditions. However, acute heat stress depressed the colonic total GPx activity which was restored to the basal level by dietary Se supplementation but not by $\alpha$-tocopherol supplementation.

Dietary Se supplementation with Se-enriched foods is an effective means to deliver Se to the population. Selenised mushrooms are an attractive food source for such a purpose in addition to selenised yeast and selenised milk proteins. However, the organic Se species profiles of yeast-Se, dairy-Se and mushroom-Se are different ${ }^{[25,31-34]}$, and there is increasing evidence that they also produce differential effect on the expression of selenoproteins relevant for Se biological activity and disease prevention such as GPx-1, GPx-2, TRx-1 and SeP $\mathrm{P}^{[22,23,26,35]}$. Selenised $A$. bisporus (button mushroom) that was cultivated by irrigation of growth compost with sodium selenite solution was used in our dietary supplementation experiment to look at the effect of the mushroom organic Se on the expression of the selenoproteins in rat colon under thermoneutral and hyperthermally induced oxidative heat stress conditions.

Rats maintained under thermoneutral conditions and fed a basic control diet supplemented with the mushroom-Se for 3 weeks significantly up-regulated colonic GPx-1 and GPx2 mRNA expression above the rats that were maintained under thermoneutral conditions but fed only the control diet. This exhibits a chronic effect of dietary mushroom-Se on increasing GPX-1 and GPx-2 expression which we have demonstrated before in the rat colon ${ }^{[26]}$. The chronic effect of Se has also been observed using different organic Se sources although there were different effects on these 2 GPx isoforms. For example, Hu, et al. ${ }^{[22]}$ showed that dietary supplementation with yeast-Se up-regulated colonic GPx-1 mRNA expression but not GPx-2 mRNA 
in the mouse, while GPx-2 mRNA expression was up-regulated by dietary supplementation with dairy-Se. In contrast, Uglietta, et al. ${ }^{[23]}$ showed that colonic GPx-2 mRNA expression was up-regulated by dietary supplementation with yeast-Se in the pig. These findings from animal studies demonstrated that the type of organic Se species present in the diet determines Se efficacy in influencing the expression of the selenoproteins. Yeast-Se occurs primarily as free selenomethionine (70-80\%) plus some free selenocysteine $(4 \%)^{[33]}$, while dairy-Se is primarily made up of free selenomethionine $(83 \%)$ and some protein bound selenocysteine $(5 \%)^{[32]}$. By contrast, our cultivated $A$. bisporus mushroom-Se used in this study consisted mainly of protein bound SeCys (95.5\%) and SeMet (3.6\%), plus a small amount of protein associated but free MeSeCys $(0.9 \%)^{[25]}$.

$\alpha$-tocopherol is known to affect the expression of some antioxidant enzymes such as GPx-4, a cell membrane associated phospholipid hydroperoxide glutathione peroxidase, whose expression level in rats is affected by $\alpha$-tocopherol levels in the $\operatorname{diet}^{[28]}$. As such, we also looked at the effect of $\alpha$-tocopherol supplementation on the expression of colonic selenoproteins under study, in rats fed the control and the mushroom-Se enriched diets. Indeed, $\alpha$-tocopherol at $300 \mu \mathrm{g} / \mathrm{g}$ feed influenced the expression of rat colonic GPxs by increasing GPx-1 but not GPx-2 mRNA levels. However, additional $\alpha$-tocopherol in the mushroom-Se/ $\alpha$-tocopherol combination diet did not produce further enhancement in the expression of colonic GPx-1 or GPx-2 in rats compared to the level induced by mushroom-Se alone, further highlighting the chronic effect of mushroom-Se on these 2 genes. Selenium in its inorganic form (sodium selenite) has also been shown to preferentially affect GPxs expression and catalase activity over $\alpha$-tocopherol in malathion challenged rats ${ }^{[36]}$.

There was an acute response in the expression of colonic GPX-1 in rats to heat stress which induced a markedly elevated expression of the gene. It is known that the adverse effect of heat stress results in the over production and accumulation of ROS in body tissues such as the intestines, which lead to oxidative stress that disrupts intestinal physiological function and reduce mucosal barrier function and integrity ${ }^{[13,19]}$. These conditions are contributing factors in the pathogenesis of gastrointestinal tract ailments such as inflammatory bowel disease, fibrosis, ulcerative colitis and colon cancer ${ }^{[1,37]}$. The elevated mRNA expression of the colonic GPx-1 in the heat stressed rats indicates an acute effect of heat stress on the colonic GPx-1 expression as the animals attempted to counter the heat induced oxidative stress in the tissue. This acute effect was observed with the animals exposed to $40^{\circ} \mathrm{C}$ heat and $21 \%$ relative humidity for only $90 \mathrm{~min}$. The expression of liver GPx-1 mRNA has also been shown to respond acutely to (cyclic) heat stress $\left(23-38-23^{\circ} \mathrm{C}\right)$ over $2 \mathrm{~h} \mathrm{du}$ rations in a similar rat strain ${ }^{[38]}$.

However, heat stressed rats fed the mushroom-Se supplemented diet exhibited an up-regulation of both colonic GPx1 and GPx-2 mRNA expression. The level of colonic GPx-1 mRNA was similar to that induced by heat stress alone for rats fed the control diet, but, significantly, the level of colonic GPX-2 mRNA was higher than that induced by heat stress alone. Supplementation with $\alpha$-tocopherol alone in the diet did not produce an enhanced expression of colonic GPx-2 observed with mushroom-Se in heat stressed rats beyond that induced by heat stress. These results supported previous observation that GI specific GPx-2 is more sensitive to changes in Se levels in the $\operatorname{diet}^{[39]}$, and additionally pointed to the larger role GPx-2 plays in the protection of the colon against hyperthermally induced oxidative stress under dietary Se supplementation. Although dietary supplementation with mushroom-Se enhanced colonic GPx-2 expression in the heat stress rats beyond that induced by heat stress alone, the combination of the mushroom-Se with $\alpha$-tocopherol in the diet failed to induce similar enhancement. It appears that the tissue required a reduced need for GPx-2 with $\alpha$-tocopherol in diet as $\alpha$-tocopherol could possibly influence the expression of non-selenium antioxidants such as catalase as the animal attempted to mitigate the effects of induced oxidative stress ${ }^{[36]}$.

Up-regulation of GPx mRNA expression in tissues normally correlates with increased GPx activity following Se dietary supplementation. Thus, both GPx-1 mRNA expression and activity were increased in mice colon following yeast-Se and dairy-Se dietary supplementation ${ }^{[11,22]}$ and in rat colon following A. bisporus mushroom-Se dietary supplementation ${ }^{[26]}$. The effect of the mushroom-Se on GPx activity was demonstrated in the present study, with an observed 3.8 fold increase in total colonic GPx activity in rats maintained under thermoneutral conditions and fed the A. bisporus mushroom-Se supplemented diet, which correlated with a 3.0 fold and 2.0 fold increased in colonic GPx-1 and GPx-2 mRNA expression, respectively. Interestingly, while rats fed the $\alpha$-tocopherol supplemented diet increased colonic GPx-1 (but not GPx-2) mRNA expression compared to rats fed the control diet, $\alpha$-tocopherol did not produced a corresponding increase in total GPx activity. It is known that GPx-1 and GPx-2 collectively are the major GPxs responsible for $70 \%$ the GPx activity in the gut detoxifying hydrogen peroxides ${ }^{[13]}$. None the less, mushroom-Se in combination with dietary $\alpha$-tocopherol supplementation produced the same effect as mushroom-Se alone in increasing colonic GPx-1 and GPx-2 mRNA expression and total colonic GPx enzyme activity.

As the adverse effect of heat stress is the over production of ROS such as hydrogen peroxide and superoxide in the body ${ }^{[13]}$, GPxs together with superoxide dismutase function as the major intracellular enzymatic antioxidant defense against $\operatorname{ROS}^{[40]}$. However, the production and removal of various ROS is a dynamic and complex process whose purpose is to attain a stable state of very low concentrations of these oxidants in the cell $^{[41]}$. Even though heat stress increased colonic GPX-1 mRNA expression in rats, it negatively impacted on colonic GPx activity level to below that of the non-heat stressed rats. Importantly, mushroom-Se supplementation in the diet restored colonic GPx activity level in heat stressed rats back to normal (that is, that of rats maintained under thermoneutral conditions and fed the control diet). In contrast, $\alpha$-tocopherol supplemented diet failed to restore colonic GPx activity level back to normal. It appears that exposure to acute heat stress might have accumulated ROS overwhelmingly in the colon that depressed GPx activity that was recovered by mushroom-Se but not by $\alpha$-tocopherol in the rats diet. With mushroom Se supplementation, the Se dependent GPxs in the tissue collectively use glutathione to detoxify organic and inorganic hydroperoxide balancing oxidative stress and associated inflammation in protecting the cells against oxidative injury ${ }^{[13]}$.

The role of TrxR is to regenerate thioredoxin from its oxidized form as part of the thioredoxin-peroxiredoxin antioxidant system that complements the glutathione peroxidase antioxidant system in the antioxidant defense of cells ${ }^{[42,43]}$. Similar 
to the GPxs, Se availability is the key factor in the expression and activity of TrxRs which included the predominant cytosolic specific TrxR-1 ${ }^{[44]}$. None the less, mushroom-Se had little or no observable effect on rat colonic TrxR-1 mRNA expression maintained under thermoneutral conditions with or without additional $\alpha$-tocopherol in diet, and $\alpha$-tocopherol itself in the diet also did not increase TrxR-1 expression. The failure of dietary $\mathrm{Se}$ in feeds in influencing GI TrxR-1 expression has been observed before in mice colon and human rectum using dairy-Se and yeast-Se ${ }^{[22,24]}$, and in rat colon using the mushroom-Se ${ }^{[26]}$ in dietary supplementations. The literature consensus is to attribute to the lesser role of the TrxR-1 in the thioredoxin-peroxiredoxin antioxidant system compared to the GPxs in the antioxidant defense of colonic and rectum tissues.

In addition to its key transport role and its influence in the expression of other selenoproteins ${ }^{[45,46]}$, SeP has also been reported to function as an antioxidant defense ${ }^{[47,48]}$. Thus, SeP knock-out mice are linked to increased carcinogen-induced cancer development ${ }^{[11,48]}$ and significant reduction or loss of $\mathrm{SeP}$ mRNA expression is observed in colorectal cancer ${ }^{[45,49]}$.

However, the literature contains contradictory reports on the effect of dietary Se on SeP expression. For example, dietary supplementation with yeast-Se has been reported to have no effect on colonic SeP mRNA expression in mice ${ }^{[22]}$ and in azoxymethane challenged rats $^{[50]}$. However, a dose dependent up-regulation of colonic SeP was observed in pigs with yeastSe, and similar to the effect on GPx-2 expression the effect was independent of sex ${ }^{[23]}$. Dairy-Se was also reported to increase colonic SeP expression in mice in a dose dependent manner ${ }^{[22]}$. In the present study, significant increase in SeP mRNA expression was observed in the colon of rats maintained under thermoneutral conditions across all supplementary diets (mushroom-Se, $\alpha$-tocopherol, mushroom-Se+ $\alpha$-tocopherol) compared to rats fed the basal diet.

Heat stress produced interesting results on the expression of TrxR-1 and SeP in rat colon across the 4 dietary regimes. Heat stress increased the expression of colonic SeP significantly but had no significant effect on TrxR-1 compared to the thermoneutral level for rats fed the control diet. Mushroom-Se produced a significant increase in the expression of both colonic SeP and TrxR-1 compared to heat stressed rats fed the control diet although expression of SeP was much higher. Furthermore, $\alpha$-tocopherol was much more effective than mushroom-Se in increasing SeP and TrxR-1 expression compared to heat stressed rats fed the control diet. But this effect was not reproduced in rats fed the mushroom-Se/ $\alpha$-tocopherol combination suggesting a reduced need for high expression of the 2 genes under heat stress, in the presence of $\alpha$-tocopherol where there is mushroom-Se.

In corroboration with our studies, it is known that $\alpha$-tocopherol influences the expression of other selenoproteins such as muscle SePx1, a selenoprotein involved in $\mathrm{ca}^{2+}$ homeostasis and schizophrenia ${ }^{[51]}$. But cells have varying prioritization of specific selenoprotein expression and synthesis in response to dietary supply of Se. For example, Se supplementation in Se deficient human subjects had GPx activity optimized before SeP concentration $^{[45]}$. Similarly, the effect of heat stress might cause this varying prioritization of specific selenoproteins expression in response to animals protecting themselves from the effects of hyperthermally induced oxidative stress. Thus, the observation of a much lower expression level of both SeP and TrxR-1 in rat colon of heat stressed rats fed the mushroom-Se/ $\alpha$-tocopherol combination diet compared to heat stressed rats fed only the $\alpha$-tocopherol diet is not surprising.

\section{Conclusions}

Se-enriched $A$. bisporus presents a viable and an efficient source of functional macro-fungal organic Se that may protect against hyperthermally induced gut injury.

The present study provided evidence that dietary supplementation with macro-fungal organic Se from Se-enriched $A$. bisporus mushroom and $\alpha$-tocopherol regulates colonic selenoproteins in rats differently under thermoneutral and heat stress conditions. GPx-1, GPx-2, TrxR-1 and SeP mRNA expression were up-regulated following dietary Se supplementation with Se-enriched $A$. bisporus under thermoneutral conditions but additional $\alpha$-tocopherol did not enhance the mushroom-Se effect. Heat stress treatment alone up-regulated GPx-1 (and not GPx2), TrxR-1 and SeP across all diets, but mushroom-Se increased GPx-2 expression above the effect of heat stress alone indicating its special role during heat stress, in the protection of the colon against hyperthermally induced gut injury. Mushroom-Se, but not $\alpha$-tocopherol, increased total colonic GPx activity in thermoneutral rats. While heat stress had a negative impact on the total colonic GPx activity, dietary supplementation with mushroom-Se, but not $\alpha$-tocopherol, recovered activity to basal (thermoneutral, control diet) level.Thus, under thermoneutral conditions Se regulated colonic selenoprotein expression with no further enhancement in selenoprotein expression with $\alpha$-tocopherol supplementation. Under hyperthermal stress conditions $\alpha$-tocopherol significantly influence selenoprotein expression particularly where Se levels are low. Where Se levels are high, Se regulate selenoprotein expression, however with no further Se effect upon $\alpha$-tocopherol supplementation.

\section{Acknowledgment}

We would like to acknowledge the contribution of $\mathrm{Mr}$. Jim Fuller (Spawn Lab Team Leader, Mushroom Exchange Pty. Ltd., 45 Cookes Road, Mernda, Victoria, Australia, 3754) in supplying the mushroom growing kits and advice on the cultivation of the $A$. bisporus, and Ms Tania Long (Animal Officer, Department of Zoology, University of Melbourne) in providing support in animal monitoring, measuring rectal temperatures and heart rates and in administering anesthesia.

\section{Conflicts of Interest}

The authors declare that there is no conflict of interests regarding the publication of this paper.

\section{References}

1. Clark, L.C., Combs, G.F. Jr., Turnbull, B.W., et al. Effects of selenium supplementations for cancer prevention in patients with carcinoma of the skin. A randomized controlled trial. Nutritional Prevention of Cancer Study Group. (1996) JAMA 276(24): 1957-1963.

2. Rayman, M.P. The importance of selenium to human health. (2000) Lancet 356(9225): 233-241.

3. Flores-Mateo, G.F., Navas-Acien, A., Pastor-Barriuso, R.,et al. Selenium and coronary heart disease; a meta-analysis. (2006) Am J Clin Nutr 84(4): 762-763.

4. Whanger, P.D. Selenocompounds in plants and animals and their bio- 
logical significance. (2002) J Am Coll Nutr21(3): 223-232.

5. Ogra, Y., Ishiwata, K., Ruiz, E.J., et al. Speciation of selenium in selenium-enriched shiitake mushroom, Lentinula edodes.(2014) Anal Bioanal Chem 379(5-6): 861-866.

6. Amoako, P.O., Uden, P.C., Tyson, J.F. Speciation of selenium dietary supplements; formation of S-(methylseleno) cysteine and other selenium compounds. (2009) Anal Chim Acta 652(1-2): 315-323.

7. Mahan, D.C., Kim, Y.Y. Effect of inorganic or organic selenium at two dietary levels on reproductive performance and tissue selenium concentrations in first-parity gilts and their progeny. (1996) J Anim Sci 74(11): 2711-2718.

8. Shiobara, Y, Yoshida, T., Suzuki, K.T. Effects of dietary selenium specieson Se concentrations in hair, blood and urine. (1998) Toxicol Appl Pharmacol 152(2): 309-314.

9. Diwadkar-Navsariwala, V., Diamond, A.M. The link between selenium and chemoprevention: A case for selenoproteins.(2004) J Nutr 134(11): 2899-2902.

10. Spolar, M.R., Schaffer, E.M., Beelman, R.B.,et al. Selenium-enriched Agaricus bisporus mushrooms suppress 7,12-dimethlybenz[a] anthracene bioactivation in mammary tissue. (1999) Cancer Lett 138(12): $145-150$.

11. Hu, Y., McIntosh, G.H., Le Leu, R.K., et al. Suppression of Colorectal Oncogenesis by Selenium-Enriched Milk Proteins: Apoptosis and K-ras Mutations. (2008) Cancer Res 68(12): 4936-4944.

12. Holben, D.H., Smith, A.M. The diverse role of selenium within selenoproteins: a review. (1999) J Am Diet Assoc 99(7): 836-843.

13. Chu, F., Esworthy, R.S., Doroshow, J.H. Role of Se-dependent Glutathione Peroxidases in Gastrointestinal Inflammation and Cancer. (2004) Free Radic Biol Med 36(12): 1481-1495.

14. Gromadzinska, J, Reszka, E, Bruzellius, K., et al. Selenium and cancer: biomarkers of selenium status and molecular action of selenium supplements. (2008) Eur J Nutr 47(Suppl 2): 29-50.

15. Lu, J., Holmgren, A. Selenoproteins. (2009) J Biol Chem 284(2): 723-727.

16. Hadley, K.B., Sunde, R.A.Selenium regulation of thioredoxin reductase activity and mRNA levels in rat liver. (2001) J Nutr Biochem 12(12): 693-702.

17. Arthur, J.R. The glutathione peroxidases. (2000) Cell Mol Life Sci 57(13-14): 1825-1835.

18. Food, Nutrition, Physical Activity, and the Prevention of Cancer: A Global Perspective. (2007) World Cancer Research Fund. American Institute for Cancer Research.

19. Pavlick, K.P., Laroux, F.S., Fuseler, J., et al. Role of reactive metabolites of oxygen and nitrogen in inflammatory bowel disease.(2002) Free Radic Biol Med 33(3): 311-322.

20. Drew, J.E., Arthur, J.R., Farquharson, A.J., et al. Salicylic acid modulates oxidative stress and glutathione peroxidase activity in the rat colon. (2005) Biochem Pharmacol 70(6): 888-893.

21. Drew, J.E., Farquharson, A.J., Arthur, J.R., et al. Novel sites of cytosolic glutathione peroxidase expression in colon. (2005)FEBS Lett 579(27): 6135-6139.

22. Hu, Y., McIntosh, G.H., Le Leu, R.K.,et al. Selenium-einriched milk proteins and selenium yeast affect selenoprotein activity and expression differently in mouse colon. (2010) Br J Nutr 104(1): 17-23.

23. Uglietta, R., Jayasooriya, S.D., Pluske, J.R., et al. Colonic expression of selenoproteins increases with level of dietary selenised yeast in finisher pig. (2007) Asia Pac J Clin Nutr 16: S70.

24. Hu, Y., McIntosh, G.H., Le Leu, R.K.,et al. The influence of selenium-enriched milk proteins and selenium yeast on plasma selenium levels and rectal selenoprotein gene expression in human subjects. (2011) Br J Nutr 106(4): 572-582.

25. Maseko, T., Callahan, D.L., Dunshea, F.R., et al. Chemical characterization and speciation of organic selenium in cultivated selenium-enriched Agaricus bisporus.(2013) Food Chem 141(4): 3681-3687.

26. Maseko, T., Howell, K., Dunshea, F.R.,et al. Selenium-enriched Agaricus bisporus increases expression and activity of glutathione peroxidase- 1 and expression of glutathione peroxidase- 2 in rat colon.
(2014) Food Chem 146: 327-333.

27. Maseko, T., Dunshea, F.R., Howell, K., et al. Selenium-Enriched Agaricus bisporus Mushroom Protects against Increase in Gut Permeability ex vivo and Up-Regulates Glutathione Peroxidase 1 and 2 in Hyperthermally-Induced Oxidative Stress in Rats. (2014) Nutrients 6(6): 2478-2492.

28. Bourre, J., Dumont, O., Clement, M., et al. Vitamin E deficiency has different effects on brain and liver phospholipid hydroperoxide glutathione peroxidase activities in the rat. (2000) Neurosci Lett 286(2): 87-90.

29. Barber, R.D., Harmer, D.W., Coleman, R.A., et al. GAPDH as a housekeeping gene: analysis of GAPDH mRNA expression in a panel of 72 human tissues. (2005) Physiol Genomics 21(3): 389-395.

30. Ellison, S.L.R., Williams, A.Eurachem/CITAC guide: quantifying uncertainty in analytical measurement.

31. Rayman, M.P. The use of high-selenium yeast to raise selenium status: how does it measure up? (2004) Br J Nutr 92(4): 557-573.

32. Heard, J.W., Stockdale, C.R., Walker, G.P., et al. Increasing Se concentration in Milk: Effects of amount of selenium from yeast and cereal grain supplements. (2007) J Dairy Sci 90(9): 4117-4127.

33. Ip, C., Birringer, M., Block, E., et al. Chemical speciation influences comparative activity of selenium-enriched garlic and yeast in mammary cancer prevention. (2000) J Agric Food Chem48(6): 2062-2070.

34. Gergely, V., Kubachka, K.M., Mounicou, S., et al. Selenium speciation in Agaricus bisporus and Lentinula edodes mushroom proteins using multi-dimensional chromatography coupled to inductively coupled plasma mass spectrometry.J Chromatogr A 1101(1-2): 94-102.

35. Uglietta, R., Doyle, P.T., Walker, G.P., et al. Bioavailability of selenium-enriched milk assessed in the artificially reared neonatal pig. (2008) Nutr Diet 65(S3): S37-S40.

36. Al-Othman, A.M., Al-Numair, K.S., El-Desoky, G.E., et al. Protection of $\alpha$-tocopherol and selenium against acute effects of malathion on liver and kidney of rat. Afr J Pharm Pharmacol 5(10): 1263-1271.

37. Kiliaan, A.J., Saunders, P.R., Bijlsma, P.B., et al. Stress stimulates transepithelial macromolecular uptake in rat jejunum. (1998) Am J Physiol 275(5 Pt 1): G1037-G1044.

38. Yung, S., Moon, Y., Sohn, S.,et al. Effects of Cyclic Heat Stress or Vitamin C Supplementation during Cyclic Heat Stress on HSP70, Inflammatory Cytokines, and the Antioxidant Defence System in Sprague Dawley Rats. (2012) Exp Anim 61(5): 543-553.

39. Banning, A., Deubel, S., Kluth, D., et al. The GI-GPx Gene is a target for Nrf2. (2005) Mol Cell Biol 25(12): 4914-4923.

40. Ganaie, A.H., Ghasura, R.S., Mir, N.A., et al. Biochemical and Physiological Changes during Thermal Stress in Bovines: A Review. (2013) Iran J Appl Anim Sci 3(3): 423-430.

41. Toussaint, O., Houbion, A., Remacle, J. Relationship between the critical level of oxidative stresses and the glutathione peroxidase activity. (1993) Toxicology 81(2): 89-101.

42. Yalcin, A. Quantification of thioredoxin mRNA expression in the rat hippocampus by real-time PCR following oxidative stress.(2004) Acta Biochim Pol 51(4): 1059-1065.

43. Zhong, L., Holmgren, A. Essential role of selenium in the catalytic activities of mammalian thioredoxin reductase revealed by characterization of recombinant enzymes with selenocysteine mutations. (2000) J Biol Chem 275(24): 18121-18128.

44. Mustacich, D., Powis, G. Thioredoxin reductase. (2000) Biochem J 346(Pt 1): 1-8.

45. Richardson, D.R. More roles for selenoprotein P: Local selenium storage and recycling protein in the brain. (2005) Biochem J 386(Pt 2): e5-e7.

46. Hoffmann, P.R., Hoge, S.C., Li, P.A., et al. The selenoproteome exhibits widely varying, tissue-specific dependence on selenoprotein $\mathrm{P}$ for selenium supply. (2007) Nucleic acids Res 35(12): 3963-3973.

47. Meplan, C., Hesketh, J. The influence of selenium and selenoprotein gene variants on colorectal cancer risk. (2012) Mutagenesis 27(2): 177-186.

48. Diwadkar-Navsariwala, V., Prins, G.S., Swanson,M.S., et al. Sele- 
noprotein deficiency accelerates prostate carcinogenesis in a transgenic model. (2006) Proc Natl Acad Sci USA 103(21): 8179-8184.

49. Al-Taie, O.H., Uceyler, N., Eubner, U., et al. Expression profiling and genetic alterations of the selenoproteins GI-GPx and SePP in colorectal carcinogenesis. (2004) Nutrition and Cancer 48(1): 6-14.

50. Uglietta, R, McIntosh, GH, Scherer, B, et al. Selenised casein increases expression of glutathione peroxidase in the colon of azoxymethane treated Sprague Dawley rat. Asia Pac J Clin Nutr 15(S 3): S77-S81. 51. Huang, J.Q., Li, D.L., Zhao, H., et al. Selenium Deficiency Disease Exudative Diathesis in Chicks Is Associated with Down regulation of Seven Common Selenoprotein Genes in Liver and Muscle. (2011) J Nutr 141(9): 1605-1610. 